\title{
Vocabulário expressivo e processamento auditivo em crianças com aquisição de fala desviante ${ }^{* * * *}$
}

\author{
Expressive vocabulary and auditory processing in children with \\ deviant speech acquisition
}

\author{
Victor Gandra Quintas* \\ Carolina Lisbôa Mezzomo** \\ Márcia Keske-Soares** \\ Roberta Freitas Dias***
}

*Fonoaudiólogo Clínico. Mestre em Distúrbios da Comunicação Humana pela Universidade Federal de Santa Maria - Rio Grande do Sul (UFSM-RS).

Endereço para correspondência: Av. Dr. Guilhermino Oliveira, 466 - Belo Horizonte - MG CEP 32341-290 (victorquintas@ymail.com).

**Fonoaudióloga. Doutora em Letras pela Pontifícia Universidade Católica do Rio Grande do Sul (PUC-RS) Professora do Curso de Graduação em Fonoaudiologia e do Programa de PósGraduação em Distúrbios da

Comunicação Humana da UFSM-RS.

***Fonoaudióloga Clínica. Mestre em Distúrbios da Comunicação Humana pela UFSM-RS.

****Trabalho Realizado no Departamento de Fonoaudiologia da UFSM-RS.

Artigo Original de Pesquisa

Artigo Submetido a Avaliação por Pares

Conflito de Interesse: não

Recebido em 06.09.2009.

Revisado em 21.02.2010; 29.04.2010; 24.07.2010; 30.07.2010.

Aceito para Publicação em 01.09.2010.

\begin{abstract}
Background: expressive vocabulary and auditory processing in children with phonological disorder. Aim: to compare the performance of children with phonological disorder in a vocabulary test with the parameters indicated by the same test and to verify a possible relationship between this performance and auditory processing deficits. Method: participants were 12 children diagnosed with phonological disorders, with ages ranging from 5 to 7 years, of both genders. Vocabulary was assessed using the ABFW language test and the simplified auditory processing evaluation (sorting), Alternate Dichotic Dissyllable - Staggered Spondaic Word (SSW), Pitch Pattern Sequence (PPS) and the Binaural Fusion Test (BF). Results: considering performance in the vocabulary test, all children obtained results with no significant statistical. As for the auditory processing assessment, all children presented better results than expected; the only exception was on the sorting process testing, where the mean accuracy score was of 8.25 . Regarding the performance in the other auditory processing tests, the mean accuracy averages were 6.50 in the SSW, 10.74 in the PPS and 7.10 in the BF. When correlating the performance obtained in both assessments, considering $\mathrm{p}>0.05$, the results indicated that, despite the normality, the lower the value obtained in the auditory processing assessment, the lower the accuracy presented in the vocabulary test. A trend was observed for the semantic fields of "means of transportation and professions". Considering the classification categories of the vocabulary test, the SP (substitution processes) were the categories that presented the higher significant increase in all semantic fields. Conclusion: there is a correlation between the auditory processing and the lexicon, where vocabulary can be influenced in children with deviant speech acquisition.
\end{abstract}

Key Words: Speech Disorders; Hearing Tests; Vocabulary.

\section{Resumo}

Tema: vocabulário expressivo e processamento auditivo em crianças com desvio fonológico. Objetivo: comparar o desempenho de crianças com desvio fonológico em teste de vocabulário com as normas apresentadas pelo mesmo e verificar a possível relação entre seus desempenhos nos testes de vocabulário e o déficit do processamento auditivo. Método: participaram da pesquisa 12 crianças com diagnóstico de desvio fonológico, com idades entre 5:0 anos e 7:0 anos e de ambos os sexos. Foi feita avaliação do vocabulário através do uso do ABFW, e avaliação simplificada do processamento auditivo (triagem), Dicótico de Dissílabos Alternados - Staggered Spondaic Word (SSW), Teste de Padrão de Frequência Pitch Pattern Sequence (PPS) e o Teste de Fusão Binaural (FB). Resultados: quanto ao vocabulário, todas as crianças obtiveram resultados sem diferenças estatisticamente significantes. Quanto ao processamento auditivo, todas as crianças tiveram resultados aquém do esperado, com exceção da triagem com média de 8,25 . Nos outros testes as médias de acerto foram, no SSW de 6,50, no PPS de 10,74 e no FB de 7,10. Ao relacionar as duas avaliações, considerando-se p >0,05, os resultados mostraram que, apesar da normalidade, quanto menor o valor obtido no processamento, menor o número de acertos apresentados no teste de vocabulário, mostrando certa tendência, principalmente nos campos "meios de transporte e profissões". Das classes, o PS (processos de substituição), foi o que obteve maior aumento significativo, sendo em todos os campos semânticos. Conclusão: há uma correlação entre processamento auditivo e o léxico, onde o vocabulário pode ser influenciado em crianças com aquisição de fala desviante.

Palavras-Chave: Vocabulário; Testes Auditivos; Distúrbios da Fala. 


\section{Introdução}

Os desvios fonológicos são alterações de fala sem etiologia orgânica aparente, mas nos quais há desordem linguística na representação mental dos sons de fala ${ }^{1}$. Este termo refere-se ao afastamento da linha de aquisição típica e não como um distúrbio, no sentido de perturbação ${ }^{2}$. As crianças normais começam a balbuciar por volta dos 6 aos 9 meses e as primeiras palavras surgem em torno dos 10 aos 15 meses $^{3}$.

É muito importante aprender o vocabulário da língua nativa, o que proporcionará integração com o meio social ${ }^{4-5}$. Contudo, a implementação semântica será possível à medida que o inventário fonológico cresce quanto ao número de fonemas. Assim, conclui-se que o desenvolvimento do léxico e da fonologia está interligado, mesmo que existam variações individuais ${ }^{6-9}$.

Somado a isto, acredita-se que as dificuldades na linguagem oral, entre elas déficit de vocabulário e fonologia, podem estar intimamente relacionadas às desordens do processamento auditivo (DPA), pois a audição é a principal via de entrada para que a aquisição lingüística seja possível ${ }^{10-13}$.

Processamento auditivo (PA) são os mecanismos e processos do sistema auditivo responsáveis pelos fenômenos comportamentais, tais como localização sonora, discriminação auditiva, reconhecimento auditivo, resolução e ordenação temporal, desempenho auditivo com sinais acústicos competitivos e/ou degradados ${ }^{13-14}$.

Apesar da importância sobre o assunto apresentado e suas implicações clínicas evidentes, há poucos estudos na literatura atual que versem sobre este tema. Assim, esta pesquisa visa investigar as prováveis relações entre o processamento auditivo e o desenvolvimento lexical em crianças com desvio fonológico.

\section{Método}

A presente pesquisa é do tipo quantitativa, transversal, de caráter descritivo-exploratório.

Este estudo foi realizado a partir de dados clínicos de crianças participantes do projeto de pesquisa "Estudo da abordagem contrastiva em três modelos de terapia fonoaudiológica para os desvios fonológicos", aprovado no Comitê de Ética em Pesquisa sob número 108/05.

Os dados foram coletados no período de setembro de 2008 a junho de 2009.

Para os sujeitos fazerem parte da amostra, foram considerados os seguintes critérios de inclusão:
. estarem autorizados pelos pais e/ou responsáveis para a participação na pesquisa por meio da assinatura do Termo de Consentimento Livre e Esclarecido e assentirem a sua participação;

- apresentarem diagnóstico de desvio fonológico (DF), ambos os sexos e idade acima de 5:0 anos, constatado durante a triagem fonoaudiológica.

Como critérios de exclusão, consideraram-se os casos de:

. alterações evidentes nos aspectos neurológico, cognitivo e/ou psicológico;

. presença de perda auditiva;

- alteração do sistema estomatognático que pudessem estar relacionados com as alterações de fala;

. realização de tratamento fonoaudiológico anterior; . dificuldade de concentração.

As crianças deste estudo foram selecionadas a partir das triagens de um serviço público de atendimento fonoaudiológico. Esta triagem permitiu perceber se havia erros na fala. O número de sujeitos na pesquisa baseou-se na demanda de atendimento fonoaudiológico apresentada na presente instituição.

As crianças que obedeciam aos critérios descritos anteriormente foram avaliadas individualmente na própria instituição e todas passaram por uma nova triagem fonoaudiológica na qual foi realizada avaliação informal da linguagem compreensiva e expressiva; avaliação fonológica completa, com o instrumento avaliação fonológica da criança $(\mathrm{AFC})^{2}$, para as crianças nas quais foi observada alteração no inventário fonológico; além das avaliações do sistema estomatognático e audiológica. Quando necessário, foram realizadas avaliações complementares neurológica, otorrinolaringológica e psicológica (caso o avaliador notasse alterações nestes níveis).

Passaram pela triagem um total de 30 crianças. Contudo, atenderam aos critérios de inclusão 18 sujeitos, com idades entre 5:0 anos e 7:0 anos e média de idade de 6:3 anos, para comporem a amostra.

Nos sujeitos da amostra, foi aplicada a avaliação de vocabulário do teste ABFW - Teste de Linguagem Infantil ${ }^{15}$, com o objetivo de verificar a competência lexical de cada criança. Por intermédio desta prova foram avaliados nove campos conceituais: vestuário, animais, alimentos, meios de transporte, móveis e utensílios, profissões, locais, formas e cores, brinquedos e instrumentos 
musicais. Essa prova analisa as designações por vocábulos usuais (DVU), as não designações (ND) e os processos de substituição (PS) utilizados pelas crianças para alcançar a nomeação correta dos vocábulos. Para cada um dos campos conceituais há uma referência de normalidade (RN). Não foi realizada entre o "Esperado" e o "Obtido", pois preocupou-se somente em analisar o processamento auditivo com o vocabulário.

Após, foi realizada a avaliação simplificada do processamento auditivo e os testes Fusão Binaural (FB) e Dicótico de Dissílabos Alternados Staggered Spondaic Word (SSW) do teste auditivo central: manual de aplicação ${ }^{16}$ e o Teste de Padrão de Frequência - Pitch Pattern Sequence (PPS) com tom puro de Musiek ${ }^{17}$. Para a aplicação de cada prova, levou-se em consideração a idade padrão em que as habilidades auditivas se desenvolveram, bem como a indicação da literatura para a aplicação de determinado teste por idade. Foi realizada uma média geral (em valores brutos) dos acertos e dos erros que poderiam ocorrer nos testes aplicados: triagem (11 respostas); FB (25 respostas); SSW (40 respostas) e PPS (30 respostas).

As avaliações, desde a triagem até as avaliações específicas nos sujeitos da amostra selecionada, foram realizadas em duas etapas, em dias diferentes. Inicialmente, foi realizada a triagem fonoaudiológica, avaliação auditiva e a avaliação fonológica para seleção da amostra. Aqueles sujeitos que estavam de acordo com os critérios de inclusão compuseram a amostra. No segundo momento, foram realizadas avaliações para a coleta dos dados, incluindo o desempenho no teste de vocabulário e a avaliação do PA*.

A análise estatística foi realizada com software SAS (Statistical Analisys System) versão 8.02, com análise de correlação de Pearson, considerando-se as correlações acima de $50 \%$ e p $<0,05$.

\section{Resultados}

Na Tabela 1 são apresentados os resultados referentes aos testes de vocabulário.

TABELA 1. Desempenho no teste de vocabulário.

\begin{tabular}{|c|c|c|c|c|c|}
\hline \multicolumn{2}{|c|}{ Teste de Vocabulário } & \multirow{2}{*}{$\begin{array}{c}\text { Média do Esperado* } \\
70,00\end{array}$} & \multirow{2}{*}{$\begin{array}{c}\text { Média do Obtido* } \\
71,67\end{array}$} & \multirow{2}{*}{$\begin{array}{c}\begin{array}{c}\text { Desvio padrão do } \\
\text { Esperado* }\end{array} \\
11,68\end{array}$} & \multirow{2}{*}{$\begin{array}{c}\begin{array}{c}\text { Desvio Padrão do } \\
\text { Obtido* }\end{array} \\
19,46\end{array}$} \\
\hline DVU & vestuário & & & & \\
\hline & animais & 61,67 & 77,83 & 11,15 & 26,41 \\
\hline & alimentos & 78,33 & 58,91 & 12,67 & 20,11 \\
\hline & meios de transporte & 65,00 & 65,16 & 10,87 & 24,45 \\
\hline & móveis e utensílios & 60,42 & 64,75 & 8,38 & 18,37 \\
\hline & profissões & 40,42 & 40,41 & 13,22 & 23,78 \\
\hline & locais & 66,67 & 45,08 & 7,78 & 29,33 \\
\hline & formas e cores & 70,83 & 68,33 & 20,32 & 22,90 \\
\hline & $\begin{array}{l}\text { brinquedos e } \\
\text { instrumentos musicais }\end{array}$ & 60,00 & 56,91 & 11,68 & 24,00 \\
\hline & média de DVU & 63,70 & 61,00 & 11,97 & 23,20 \\
\hline \multirow{10}{*}{$\mathrm{ND}$} & vestuário & 3,33 & 0,00 & 3,89 & 0,00 \\
\hline & animais & 18,33 & 2,83 & 2,46 & 7,87 \\
\hline & alimentos & 10,83 & 7,75 & 6,33 & 11,61 \\
\hline & meios de transporte & 3,33 & 4,50 & 2,46 & 9,00 \\
\hline & móveis e utensílios & 5,00 & 3,33 & 0 & 4,77 \\
\hline & profissões & 26,67 & 1,67 & 4,44 & 3,89 \\
\hline & locais & 10,00 & 2,08 & 7,38 & 5,23 \\
\hline & formas e cores & 10.83 & 3,33 & 12,58 & 4,92 \\
\hline & $\begin{array}{l}\text { brinquedos e } \\
\text { instrumentos musicais }\end{array}$ & 17.67 & 7,50 & 20,62 & 12,03 \\
\hline & média de ND & 11,07 & 3,66 & 6,68 & 6,59 \\
\hline \multirow[t]{10}{*}{ PS } & vestuário & 26,67 & 24,17 & 7,78 & 10,83 \\
\hline & animais & 20,00 & 19,33 & 11,68 & 23,70 \\
\hline & alimentos & 10,83 & 29,08 & 6,34 & 7,92 \\
\hline & meios de transporte & 33,33 & 29,33 & 8,88 & 23,96 \\
\hline & móveis e utensílios & 30,00 & 29,41 & 8,26 & 17,81 \\
\hline & profissões & 35,00 & 56,67 & 5,22 & 22,69 \\
\hline & locais & 23,33 & 51,67 & 2,46 & 26,39 \\
\hline & formas e cores & 21,67 & 25,00 & 18,50 & 21,95 \\
\hline & $\begin{array}{l}\text { brinquedos e } \\
\text { instrumentos musicais }\end{array}$ & 28,33 & 35,33 & 8,88 & 23,73 \\
\hline & média de PS & 25,46 & 33,33 & 8,67 & 19,88 \\
\hline
\end{tabular}

Legenda: DVU - designação por vocábulo usual; ND - não designação; PS - processo de substituição; * valores absolutos - dependendo do comportamento e disposição do paciente, esta avaliação foi dividida entre dois dias (dias correntes). 
Tratando-se da DVU, nota-se que a média geral esperada é de 63,7 , e a média geral obtida foi de 61,0 , havendo uma pequena diferença entre eles. Os campos conceituais alimentos, profissões, locais, formas e cores e brinquedos e instrumentos musicais obtiveram escores abaixo do esperado (Tabela 1).

Quanto ao ND, a classe obteve uma média geral de 3,66, sendo o esperado de 11,07. Apenas o campo conceitual meios de transporte obteve escore acima do esperado (Tabela 1 ).

Por fim, nos casos de o PS, a média geral obtida foi de 33,33 , sendo superior à média esperada de 25,46 . Os campos conceituais alimentos, profissões, locais, formas e cores e brinquedos e instrumentos musicais obtiveram escores maiores do que o esperado (Tabela 1).

Quanto ao PA, temos a média de acertos e erros de acordo com a possibilidade de cada teste, desta forma, no teste Triagem temos que o valor máximo de acerto seria 11; no teste SSW temos 40; no teste PPS temos 30; e no teste FB temos 25. Foram considerados alterados os testes que apresentaram valores de média de erros próximo do valor total possível (Tabela 2).

A Tabela 3 mostra as correlações que se mostraram significantes entre o desempenho nas tarefas de vocabulário e nos testes do PA.

TABELA 2. Desempenho nos testes do processamento auditivo.

\begin{tabular}{c|c|c|c|c}
\hline Teste & Média dos Acertos* & $\begin{array}{c}\text { Desvio Padrão dos } \\
\text { Acertos* }\end{array}$ & Média dos Erros* & $\begin{array}{c}\text { Desvio Padrão dos } \\
\text { Erros* }\end{array}$ \\
\hline triagem & 8,25 & 2,90 & 3,00 & 3,16 \\
SSW & 6,50 & 7,15 & 33,50 & 7,14 \\
PPS & 10,74 & 6,54 & 19,25 & 6,56 \\
fusão binaural & 7,10 & 2,76 & 17,90 & 2,74 \\
\hline
\end{tabular}

Legenda: SSW - Staggered Spondaic Word (dissílabos alternados); PPS - Pitch Pattern Sequence (padrão de frequência); FB - fusão binaural. Consideraram-se erro todas as respostas que estejam em desacordo com a normalidade; * valores brutos.

TABELA 3. Correlação entre o processamento auditivo e o vocabulário de crianças com desvio fonológico.

\begin{tabular}{c|l|c|c|c|c}
\hline Classes & \multicolumn{1}{c|}{ Campo Conceitual } & Triagem & SSW & PPS & FB \\
\hline \multirow{2}{*}{ DVU } & animais & 0,63 & - & - & - \\
& alimentos & 0,64 & - & - & - \\
& meios de transporte & 0,67 & - & - & - \\
& profissões & 0,58 & 0,54 & 0,64 & - \\
& locais & - & - & - & - \\
& formas e cores & 0,52 & - & - & - \\
& brinquedos e instrumentos musicais & - & - & - & - \\
\hline \multirow{2}{*}{ ND } & meios de transporte & $-0,66$ & - & - & $-0,84$ \\
& locais & - & - & - & - \\
\hline \multirow{2}{*}{ OS } & animais & $-0,59$ & - & - & - \\
& alimentos & - & $-0,72$ & $-0,56$ & - \\
& meios de transporte & - & - & $-0,50$ & - \\
& profissões & $-0,60$ & $-0,50$ & $-0,64$ & - \\
\hline
\end{tabular}

Legenda: DVU - designação por vocábulo usual; ND - não designação; PS - processo de substituição. Foram tabelados apenas os valores de correlação $\geq 0,50$ e p $<0,05$. Teste estatístico utilizado: Correlação de Pearson. 
Ao relacionar o desempenho nas tarefas de vocabulário e os testes do PA observa-se uma correlação positiva entre a DVU e o resultado nos testes de processamento, ou seja, quanto melhor o desempenho no PA melhor é a designação por vocabulário usual. Esta correlação foi realizada entre os acertos do processamento e a porcentagem de DVU. Tem-se que a triagem possui a maior correlação com os campos conceituais, na classe DVU, apresentando valores significativos e acima ou igual à 0,50 em cinco campos. O teste SSW apresenta correlação apenas com um único campo conceitual na DVU; O PPS apresenta correlação com dois campos, e por fim o teste FB possui correlação, também, com apenas um campo conceitual naDVU(Tabela3).

Quanto à classe ND, tem-se que apenas a triagem e oFB têm correlação com algum dos campos conceituais. Tais correlações apresentam-se negativas, o que indica que, quanto pior os sujeitos se apresentaram nos testes de processamento, mais não designações houve nestes campos conceituais (Tabela 3 ).

A mesma correlação negativa está presente em PS. A triagem e o SSW possuem esta correlação com dois campos conceituais e o PPS com quatro campos, sendo este o que mais pode influenciar esta classe. $O$ teste FB não apresentou qualquer correlação. A correlação negativa nesta classe mostra que, quanto pior o desempenho nos testes apresentados, mais processos de substituição estarão presentes (Tabela 3).

\section{Discussão}

Os resultados desta pesquisa estão de acordo com a hipótese de que crianças com DF apresentam DPA, mas que o nível lexical pode não estar alterado. Durante a evolução da linguagem, o desenvolvimento do léxico e da fonologia está interligado, mesmo existindo variações individuais ${ }^{6}$. Entretanto, como visto nesta pesquisa, um pode não influenciar o outro.

Os resultados apresentados sobre o desempenho no vocabulário das crianças com DF estão de acordo com parte da literatura, que mostra não haver diferença significativa entre as crianças com aquisição de fala desviante e as crianças com aquisição normal ${ }^{4,18-19}$. Entretanto, não concorda com outros autores que sugerem haver alteração lexical em crianças com $\mathrm{DF}^{7,20}$.

Entre todos os campos conceituais, o campo Meio de Transporte foi o único que apresentou correlação estatisticamente significativa em todas as três classes, DVU, ND e PS, entretanto, o campo Profissões foi o que mais mostrou ser influenciado pelo baixo desempenho nas tarefas do PA (Tabela 3). Vale ressaltar que todos os sujeitos desta pesquisa apresentam DF.
Com exceção da triagem, nota-se desempenho baixo nos testes do PA realizados nas crianças deste estudo, o que está de acordo com as hipóteses desta pesquisa, onde crianças com desvio fonológico pode apresentar alteração no PA.

Quando o sinal acústico é degradado ou existe um sinal acústico competitivo, o DPA pode afetar a compreensão, a fala, a escuta e o aprendizado ${ }^{21-22}$. Considerando dois estudos ${ }^{23-24}$ e a presente pesquisa, tal fato pode ser demonstrado, uma vez que os resultados quanto ao vocabulário, embora não sejam significativos, estão de acordo com o desempenho nos testes do processamento.

O desempenho geral do vocabulário, principalmente na classe DVU, mostra que seu bom desempenho pode estar ligado a escores altos também na avaliação simplificada do processamento. Já o teste FB é o que possui pior resultado, o que demonstra ser este teste o de maior dificuldade para as crianças.

Além disso, o resultado do PA nestas crianças com desvio fonológico permite dizer que ambos estariam intimamente correlacionados. Este dado é corroborado por alguns estudos ${ }^{7,25-27}$. Entretanto, não foi realizada análise deste achado, uma vez que não se tratava do objetivo da pesquisa.

A relação PA e aquisição lexical é muito pouco explorada, mesmo em crianças com desenvolvimento de fala normal, contudo, alguns estudos que tratam da aquisição de linguagem, abordam o vocabulário ${ }^{5,7,11-13,22-23,29-30}$.

Entretanto, é importante destacar que, clinicamente, o desvio fonológico pode estar presente em crianças com DPA, mas o número de palavras presente no vocabulário destes sujeitos pode não estar alterado, desta forma, durante a clínica da fala, há possibilidade de maior gama de palavras a serem usadas no tratamento destas crianças com alteração de PAe DF. Contudo, novas pesquisas permitiram confirmar estes achados.

O bom desenvolvimento das habilidades auditivas, bem como as estruturas relacionadas, é de estrema importância para a aquisição da fala e do léxico ${ }^{5,10-12,23,27,29}$.

\section{Conclusão}

Este trabalho mostrou que crianças com desvios fonológico podem apresentar DPA, mas que o vocabulário destas crianças não está tão deficitário quanto se esperava. Entretanto, aquisição lexical e PA apresentam-se relacionados entre si nesta população, evidenciando que quanto melhor estiver o resultado em avaliações do processamento, melhor o desempenho em vocabulário expressivo. 


\section{Referências Bibliográficas}

1. Lewis BA, Freebairn LA, Hansen AJ, Stein CM, Shriberg LD, Iyengar SK, Taylor HG. Dimensions of early speech sound disorders: A factor analytic study. J Comm Disorders. 2006;39:139-57.

2. Yavas MS, Hernandorena CLM, Lamprecht RR. Avaliação fonológica da criança: reeducação e terapia. Porto Alegre: Artes Médicas; 1991.

3. Wertzner HF, Amaro L, Galea DES. Phonological performance measured by speech severity indices compared with correlated factors. Sao Paulo Med J. 2007;125(6):30914.

4. Gathercole SE, Baddeley AD. Evaluation of the role of phonological STM in the development of vocabulary in children: A longitudinal study. Journal of Memory and Language. 1989;28:200-13.

5. Tallal P. Auditory temporal perception, phonics and reading disabilities in children. Brain Lang. 1980;9:18298 .

6. Stoel-Gammon C. Normal and disordered phonology in two-years-olds. Topic in language disorders. 1991;11(4):2132 .

7. Araújo MR, Minervino CASM. Avaliação cognitiva: leitura, escrita e habilidades relacionadas. Psicologia em Estudo. 2008;13(4):859-65.

8. Hage SRV, Pereira MB. Desempenho de crianças com desenvolvimento típico de linguagem em prova de vocabulário expressivo. Revista CEFAC. 2006;8(4):41928 .

9. McCune L, Vihman MM. Early phonetic and lexical development: A productivity approach. J Speech Lang Hear Res, 2001;44:670-84.

10. Chermak GD, Silva ME, Nye J, Hasbrouck J, musiek FE. An update on professional education and clinical practices in central auditory. Journal of the American Academy of Audiology. 2007;18(5):428-52.

11. Bamiou DE, Musiek FE, Luxon LM. Aetiology and clinical presentations of auditory processing disorders- a review. Arch Dis Child. 2001;85:361-5.

12. Bishop DV, Carlyon RP, Deeks JM, Bishop SJ. Auditory temporal processing impairment: neither necessary nor sufficient for causing language impairment in children. J Speech Lang Hear Res. 1999;42(6):1295-310

13. Psillas G, Psifidis A, Hitoglou-Antoniadou M, Kouloulas A. Hearing assessment in pre-school children with speech delay. Auris Nasus Larynx. 2006;33:259-63.

14. Cacare AT, McFarland DJ. The importance of Modality Specificity in Diagnosing Central Auditory Processing Disorder. American Journal of Audiology. 2005;14:112-23

15. Befi-Lopes DM. Vocabulário. In: Andrade CRF, BefiLopes DM, Fernandes FDM, Wertzner HF. Teste de linguagem infantil: nas áreas de fonologia, vocabulário, fluência e pragmática. Carapicuíba: Pró-Fono; 2000.
16. Pereira LD, Schochat E. Processamento Auditivo Central: manual de aplicação. São Paulo: Lovise, 1997.

17. Auditec. Evaluation manual of pitch pattern sequence and duration pattern sequence. Missouri, 1997.

18. Athayde ML. Vocabulário expressivo e habilidades de memória de trabalho em crianças com desenvolvimento fonológico normal e desviante [Dissertação]. Santa Maria (RS): Universidade Federal de Santa Maria; 2009.

19. Befi-Lopes DM, Gândara JP. Desempenho em prova de vocabulário de crianças com diagnóstico de alteração fonológica. Rev da Soc Bras de Fonoaudiologia. 2002;7(1):16-23.

20. Mota HB, Kaminski TI, Nepomuceno MRFN, Athayde ML. Alterações no vocabulário expressivo de crianças com desvio fonológico. Rev Soc Bras Fonoaudiol. 2009;14(1):41-7.

21. Sharma M, Purdy SC, Kelly AS. Comorbidity of Auditory Processing, Language, and Reading Disorders. Journal of speech language and hearing research. 2009; 52(3):70622.

22. Murphy CFB e Schochat E. How auditory temporal processing deficits relate to dyslexia. Brazilian Journal of Medical and Biological Research. 2009;42:647-54.

23. American Speech-Language-Hering Association. Central auditory processing: current status of reaserch and implications of clinical practice. American Journal Audiology. 1996;5.

24. Wohllben B, Rosenfeld J, Gross M. Auditive Verarbeitungsund Wahrnehmungsstörungen (AVWS). Phoniatrie und Pädaudiologie 2007;55:403-10.

25. Heath SM, Hogben JH, Clark CD. Auditory temporal processing in disabled readers with and without oral language delay. J Child Psychol Psychiatry. 1999;40(4):637-47.

26. Boets B, Wouters $\mathrm{J}$, van Wieringen $\mathrm{A}$, Ghesquière $\mathrm{P}$. Auditory processing, speech perception and phonological ability in pre-school children at high-risk for dyslexia: A longitudinal study of the auditory temporal processing theory. Neuropsychologia. 2007;45(8):1608-20.

27. Roggia SM. O processamento temporal em crianças com desvio fonológico [Tese]. São Paulo(SP): Faculdade de Medicina da Universidade de São Paulo; 2003.

28. Samelli AG, Schochat E. Processamento auditivo, resolução temporal e teste de detecção de gap: revisão da literatura. Revista CEFAC. 2008;10(3):369-77.

29. Engelmann L, Ferreira MIDC. Avaliação do processamento auditivo em crianças com dificuldades de aprendizagem. Rev Soc Bras Fonoaudiol. 2009;14(1):6974.

30. Tallal P, Miller S, Fitch RH. Neurobiological basis of speech: a case for the preeminence of temporal processing. Ann N Y Acad Sci. 1993;682:27-47. 